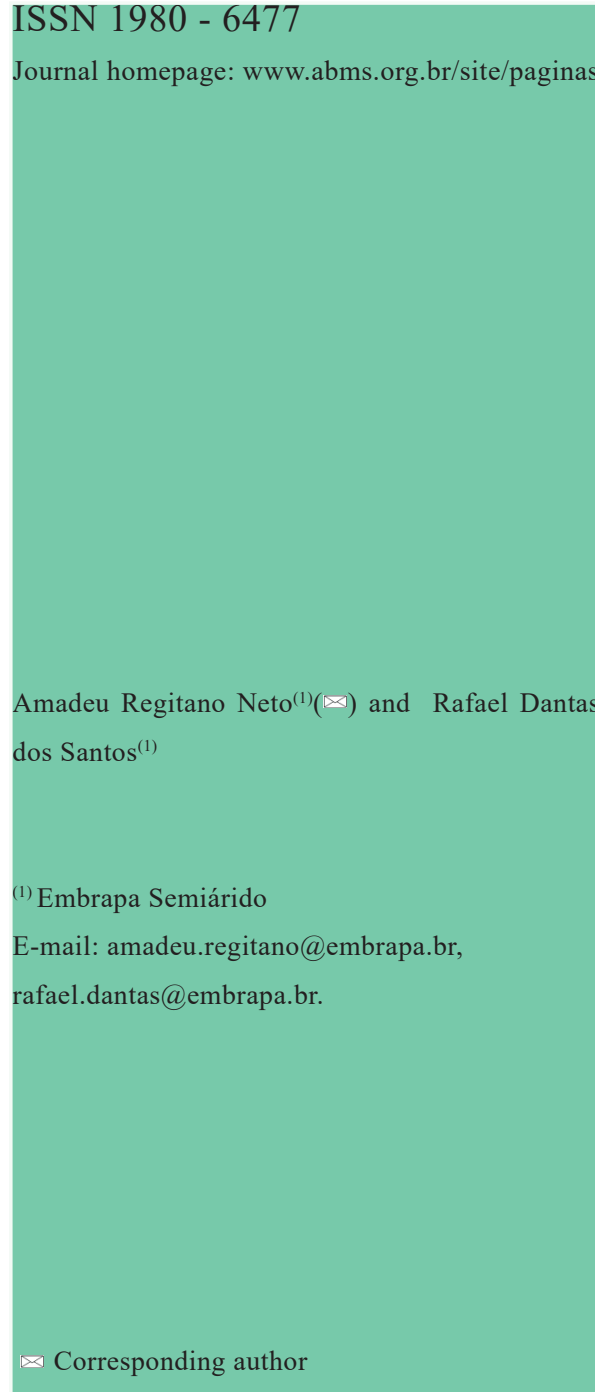

How to cite

REGITANO NETO, A.; SANTOS, R. D. Agronomic traits evaluation in irrigated sorghum in the brazilian semi-arid. Revista Brasileira de Milho e Sorgo, v. 19, e1179, 2020.

\section{AGRONOMIC TRAITS EVALUATION IN IRRIGATED SORGHUM IN THE BRAZILIAN SEMI-ARID}

\begin{abstract}
Green and dry matter production, along with grain yield and other agronomic traits, were assessed in 44 sorghum genotypes. Two sets of genotypes were formed based on known plant height, aiming to evaluate green and dry matter production and grain yield, as well as to separate forage and grain sorghum genotypes. The evaluations were performed based on experiments with three replications, being one irrigated (drip system) in Petrolina, State of Pernambuco, and the other rainfed, in Nossa Senhora da Glória, state of Sergipe, Brazil. Sowing dates were July 30, 2016, in Nossa Senhora da Glória; July 13, 2017, and October 24, 2018, in Petrolina; with 117 and 128 days from sowing to harvest, respectively. Expressive forage production was observed in genotypes EP-17 and SF-11, which exceeded $120 \mathrm{t} \mathrm{ha}^{-1}$. BRS 506 produced $108 \mathrm{t} \mathrm{ha}^{-1}$, while 13F03(1141572), P-294, P-288, 2502 x 467, BRS Ponta Negra, and SF-15 presented green matter production ranging from 94 to $98.5 \mathrm{t} \mathrm{ha}^{-1}$. Dry matter production was highly correlated with green matter production, and SF-11 had the best performance of $45.5 \mathrm{t} \mathrm{ha}^{-1}$. The observed plant height was more expressive for forage sorghum genotypes, being this trait highly correlated with the green and dry matter. The best grain yield performance reached 13.4 and $10.3 \mathrm{t} \mathrm{ha}^{-1}$, values observed for 9910032 and BRS Ponta Negra, respectively. The results demonstrate the full adaptability of sorghum to the semi-arid environment and the feasibility to produce roughage and grains under irrigation in the Brazilian semi-arid region.
\end{abstract}

Keywords: Sorghum bicolor, roughage, forage, grain yield, caatinga.

\section{AVALIAÇÃO DE CARACTERÍSTICAS AGRONÔMICAS DE SORGO IRRIGADO NO SEMIÁRIDO BRASILEIRO}

Resumo - A produção de matéria verde e seca juntamente com a produção de grãos e outros caracteres agronômicos foram avaliados de 44 genótipos de sorgo. Dois conjuntos de genótipos foram formados com base na altura de planta conhecida, com o objetivo de avaliar a produção de grãos e de biomassa verde e seca além de separar materiais forrageiros de graníferos. Avaliações foram feitas em experimentos com três repetições com irrigação por gotejamento em Petrolina, PE e dependente de chuva em Nossa Senhora da Glória, SE. Em Nossa Senhora da Glória o plantio ocorreu em 30 de julho de 2016, e em Petrolina, em 13 de julho de 2017 e 24 de outubro de 2018, com 117 e 128 dias de ciclo, respectivamente. Expressiva produção de forragem foi observada, superior à $120 \mathrm{t} \mathrm{ha}^{-1}$ no genótipo EP-17 e SF-11, enquanto BRS 506 produziu $108 \mathrm{t} \mathrm{ha}^{-1}$ e 13F03(1141572), P-294, P-288, 2502 x 467, BRS Ponta Negra e SF-15 produziram matéria verde no intervalo entre $94 \mathrm{e} 98,5 \mathrm{tha}^{-1}$. A produção de matéria seca foi altamente correlacionada com a produção de matéria verde e a melhor performance foi $45,5 \mathrm{t} \mathrm{ha}^{-1}$ para SF-11. A altura de plantas observada foi mais expressiva para os tipos forrageiros e esse caráter foi altamente correlacionado com matéria verde e seca. A melhor performance em produção de grãos alcançou 13,4 e 10,3 tha ${ }^{-1}$, sendo observada em 9910032 e BRS Ponta Negra, nessa ordem. Os resultados obtidos demonstram a total adaptação do sorgo ao ambiente semiárido e a viabilidade de produzir volumoso e grãos sob irrigação no semiárido brasileiro.

Palavras-chave: Sorghum bicolor, volumoso, forragem, produção de grãos, caatinga. 
Animal farming in semi-arid environment is usually associated with uncertainties regarding water availability for animals and crops. In Brazil, the caatinga environment comprises around 980 thousand square kilometers and is one of the most populated dry areas in the world, being a home to more than 22 million people, although having to live with low socioeconomic indices (Silva et al., 2010).

Livestock farming has been the main activity for most farmers in the caatinga, and a large amount of sheep (12.6 million) and goats (10.0 million) are been reared in the 9 states of the northeastern part of Brazil. Moreover, 27.8 million bovine animals are raised in this environment, distributed over less dry areas, aimed at dairy farming (IBGE, 2019).

Forage production is a difficult task in the semi-arid climate and irrigation plays a major role in enabling crop production under such a restrictive environmental condition.

Sorghum (Sorghum bicolor L. Moench), along with other forage grasses, is a highly promising crop for animal feeding, and produces a large amount of high quality green forage that can be either served chopped to animals or stored, preserved in form of silage, which represents a very feasible strategy for animal consumption during warmer and drier months.

With origins dating back 5 or 6 thousand years, where we can find Sudan and Ethiopia in the African continent (Santos et al., 2005), sorghum accumulated, through generations of natural selection, characteristics to grow and produce seeds under that sub-Saharan environment. Such drought resistance traits include C4 photosynthetic pathway, usual in plants from hot and arid places subject to drought; stomates on both sides of the leaves; ability to roll the leaves lengthwise creating a more secure microenvironment to open the stomates; and a wax deposition in the foliar limbus - leaf sheath junction (Magalhães et al., 2003).

Sorghum producers can find several hundreds of registered sorghum cultivars in the Brazilian seed market, most of them developed for grain production, though forage and double purpose (grain / forage) cultivars are also available and of major interest for northeastern livestock producers.

This report presents green and dry matter yield and other biometric data for 44 sorghum genotypes cultivated under drip irrigation, in the caatinga environment of the São Francisco River Valley in Petrolina, state of Pernambuco, and under rainfed conditions, in the agreste environment of Nossa Senhora da Glória, state of Sergipe, Brazil.

\section{Material and Methods}

Performance analyses took place at the Experimental Field of Nossa Senhora da Glória (C.E.G.), state of Sergipe, in 2016 (Environment 1), and the Experimental Field of Bebedouro (C.E.B.), from Embrapa Semi-Arid in Petrolina, state of Pernambuco, in 2017 (Environment 2) and 2018 (Environment 3), all in the northeast 
region of Brazil. A randomized block design was used for the experiment, with 3 replications. The experimental plots consisted of 4 rows of 5 meters, spaced $0.8 \mathrm{~m}$ apart, with 15 plants $\mathrm{m}^{-1}$. Drip irrigation was available and water was applied as needed. For measurements, only the two central rows were considered as useful area, discarding $0.5 \mathrm{~m}$ from each end. Fertilizer was applied based on soil analysis, and before the 2017 and the 2018 crops at C.E.B. The analysis revealed the following characteristics: $\mathrm{pH}=6.10$; $\mathrm{P}=28.43 \mathrm{mg} \mathrm{dm}^{-3} ; \mathrm{K}=0.38 \mathrm{cmol}_{\mathrm{c}} \mathrm{dm}^{-3} ; \mathrm{Al}=0.10$ $\mathrm{cmol}_{\mathrm{c}} \mathrm{dm}^{-3} ; \mathrm{H}+\mathrm{Al}=1.71 \mathrm{cmol}_{\mathrm{c}} \mathrm{dm}^{-3} ; \mathrm{Ca}=1.70$ $\mathrm{cmol}_{\mathrm{c}} \mathrm{dm}^{-3} ; \mathrm{Mg}=2.00 \mathrm{cmol}_{\mathrm{c}} \mathrm{dm}^{-3} ; \mathrm{C}=0.00 \mathrm{~g} \mathrm{~kg}^{-1}$; $\mathrm{V}=71.0 \%$, which determined sowing fertilization with $300 \mathrm{~kg} \mathrm{ha}^{-1}$ of the 6-24-12 formula and top dressing with $70 \mathrm{~kg} \mathrm{ha}^{-1}$ of nitrogen, divided in three applications at 15,30 and 45 days after emergence. Sowing dates were July 30, 2016 for Environment 1; July 13, 2017 for Environment 2; and October 24, 2018 for Environment 3. Harvest occurred at 117 days for Environment 2 and 128 days for Environment 3.

The evaluated genotypes are listed in Table 1 and comprised commercial cultivars from the seed market, experimental populations derived from institutional breeding programs (Embrapa and IPA - Agricultural and Livestock Research Enterprise of the State of Pernambuco), and accessions from the sorghum germplasm bank of Embrapa Maize and Sorghum. The genotypes were divided into two groups, based on the known plant height. In Experiment 1, taller plants were included, indicating types of cultivars for forage production, while Experiment 2 comprised the shorter plants, with assumed grain production ability. Evaluation was carried out for each genotype group formed in an individual experiment, with 6 common genotypes, identified in Table 1, used as checks.

Evaluations were performed and biometric data were taken from the essays. Information of flowering (FLO, days), plant height (PH, m) and panicle length $(\mathrm{PL}, \mathrm{cm})$ were collected in Environment 1; flowering, plant height, green matter yield (GM, t ha $\left.{ }^{-1}\right)$, dry matter yield (DM, $\mathrm{t} \mathrm{ha}^{-1}$ ), grain yield (GY, $\left.\mathrm{kg} \mathrm{ha}^{-1}\right)$ and seed mass (SM, mg) in Environment 2; and plant height and green matter yield in Environment 3.

Simple and joint analysis of variance (ANOVA) were performed, and Scott-Knott mean test $(p<0.05)$ was used to rank the results. Pearson's correlation coefficients were estimated between the traits.

\section{Results and Discussion}

The variability observed among sorghum genotypes in the experiments was very wide. Even considering one experiment at a time, a large variation could be observed for matter production (green or dry) and plant architecture.

From the experiments with forage plants (Table 2), the most expressive GM production exceeded $120 \mathrm{t} \mathrm{ha}^{-1}$ for genotypes EP-17 and SF-11. BRS 506 produced $108 \mathrm{t} \mathrm{ha}^{-1}$, while 13F03(1141572)，P-294，P-288， 2502 x 467, BRS Ponta Negra and SF-15 were in the range 
Table 1. Sorghum genotypes evaluated in Petrolina, PE, and in Nossa Senhora da Glória, SE.

\begin{tabular}{|c|c|c|c|}
\hline Number (\#) & Genotypes (G) & $\begin{array}{l}\text { Experiment } \\
\text { (E) }\end{array}$ & Institution \\
\hline 1 & BRS 506 & 1 & Embrapa \\
\hline 2 & 13F03(1141562) & 1 & Embrapa \\
\hline 3 & 13F03(1141572) & 1 & Embrapa \\
\hline 4 & 947216 & 1 & Embrapa \\
\hline 5 & 13F02(1141570) & 1 & Embrapa \\
\hline 6 & 9929030 & 1 & Embrapa \\
\hline 7 & 947030 & 1 & Embrapa \\
\hline 8 & 947254 & 1 & Embrapa \\
\hline 9 & SS3 18 & 1 & Embrapa \\
\hline 10 & ICSV745 & 1 & Embrapa \\
\hline 11 & IPA 2502 & 1 & IPA \\
\hline 12 & EP-17 & 1 & IPA \\
\hline 13 & SF-11 & 1 & IPA \\
\hline 14 & P-294 & 1 & IPA \\
\hline 15 & P-288 & 1 & IPA \\
\hline 16 & BRS 506 X 2506 & 1 & IPA \\
\hline 17 & $2502 \times 467$ & 1 & IPA \\
\hline 18 & Theis X 2501 & 1 & IPA \\
\hline 19 & IPA 2564 & 1 & IPA \\
\hline 20 & Sureno 160.0 & 2 & Embrapa \\
\hline 21 & ICSV400 & 2 & Embrapa \\
\hline 22 & EBA-3 & 2 & Embrapa \\
\hline 23 & SC971 & 2 & Embrapa \\
\hline 24 & FC13642 Pink Kafir (ASA N²2) & 2 & Embrapa \\
\hline 25 & Marupantse & 2 & Embrapa \\
\hline 26 & PI48770 White Kafir (ASA N²1) & 2 & Embrapa \\
\hline 27 & M35-1 & 2 & Embrapa \\
\hline 28 & $\mathrm{SC} 224$ & 2 & Embrapa \\
\hline 29 & SC1337 & 2 & Embrapa \\
\hline 30 & SC323 & 2 & Embrapa \\
\hline 31 & Segaolane & 2 & Embrapa \\
\hline 32 & Sureno 167.5 & 2 & Embrapa \\
\hline 33 & CMSXS189 & 2 & Embrapa \\
\hline 34 & $\mathrm{SC} 720$ & 2 & Embrapa \\
\hline 35 & 9910032 & 2 & Embrapa \\
\hline 36 & Pinolero 1 & 2 & Embrapa \\
\hline 37 & Jocoro & 2 & Embrapa \\
\hline 38 & Kuyuma & 2 & Embrapa \\
\hline 39 & SF-15 & check & IPA \\
\hline 40 & BRS Ponta Negra & check & Embrapa \\
\hline 41 & BRS 655 & check & Embrapa \\
\hline 42 & Volumax & check & Monsanto \\
\hline 43 & BRS 658 & check & Embrapa \\
\hline 44 & BRS 659 & check & Embrapa \\
\hline
\end{tabular}


Table 2. Means of green matter and plant height for 25 forage sorghum genotypes (E1) evaluated in Petrolina, PE (C.E.B) and in Nossa Senhora da Glória, SE (C.E.G.).

\begin{tabular}{|c|c|c|c|c|c|c|c|c|c|c|}
\hline \multirow{3}{*}{$\begin{array}{l}\text { Genotypes } \\
\text { BRS } 506\end{array}$} & \multicolumn{4}{|c|}{ Green Matter $\left(\mathrm{t} \mathrm{ha}^{-1}\right)^{*}$} & \multicolumn{6}{|c|}{ Plant Height $(\mathrm{m})$} \\
\hline & \multicolumn{2}{|c|}{ C.E.B. 2017} & \multicolumn{2}{|c|}{ C.E.B 2018} & \multicolumn{2}{|c|}{ C.E.B. 2017} & \multicolumn{2}{|c|}{ C.E.B 2018} & \multicolumn{2}{|c|}{ C.E.G 2016} \\
\hline & 108.1 & $\mathrm{Aa}$ & 59.6 & $\mathrm{Bb}$ & 2.22 & $\mathrm{Bb}$ & 2.03 & $\mathrm{Bb}$ & 2.76 & $\mathrm{Aa}$ \\
\hline 13F03(1141562) & 94.0 & $\mathrm{Aa}$ & 85.4 & $\mathrm{Aa}$ & 1.83 & $\mathrm{Bc}$ & 3.08 & $\mathrm{Aa}$ & 2.41 & $\mathrm{Ba}$ \\
\hline 13F03(1141572) & 56.2 & $\mathrm{Ab}$ & 76.2 & $\mathrm{Aa}$ & 1.47 & $\mathrm{Bc}$ & 2.25 & $\mathrm{Ab}$ & 2.57 & $\mathrm{Aa}$ \\
\hline 947216 & 76,2 & $\mathrm{Ab}$ & 62.3 & $\mathrm{Ab}$ & 1.49 & $\mathrm{Bc}$ & 1.99 & $\mathrm{Bb}$ & 2.56 & $\mathrm{Aa}$ \\
\hline 13F02(1141570) & 88.5 & $\mathrm{Aa}$ & 80.8 & $\mathrm{Aa}$ & 2.26 & $\mathrm{Ab}$ & 2.92 & $\mathrm{Aa}$ & 2.35 & $\mathrm{Aa}$ \\
\hline 9929030 & 65.9 & $\mathrm{Ab}$ & 54.8 & $\mathrm{Ab}$ & 1.09 & $\mathrm{Bc}$ & 1.69 & $\mathrm{Ab}$ & 1.93 & $\mathrm{Aa}$ \\
\hline 947030 & 58.0 & $\mathrm{Ab}$ & 49.5 & $\mathrm{Ab}$ & 1.57 & $\mathrm{Bc}$ & 1.73 & $\mathrm{Bb}$ & 3.06 & $\mathrm{Aa}$ \\
\hline 947254 & 63.2 & $\mathrm{Ab}$ & 51.5 & $\mathrm{Ab}$ & 1.63 & Ac & 2.17 & $\mathrm{Ab}$ & 2.28 & $\mathrm{Aa}$ \\
\hline SS318 & 73.3 & $\mathrm{Ab}$ & 63.7 & $\mathrm{Ab}$ & 1.62 & $\mathrm{Bc}$ & 2.17 & $\mathrm{Ab}$ & 2.43 & $\mathrm{Aa}$ \\
\hline ICSV745 & 61.3 & $\mathrm{Ab}$ & 62.1 & $\mathrm{Ab}$ & 1.89 & $\mathrm{Bc}$ & 2.59 & $\mathrm{Aa}$ & 2.85 & $\mathrm{Aa}$ \\
\hline IPA 2502 & 38.2 & $\mathrm{Bb}$ & 46.7 & $\mathrm{Ab}$ & 1.36 & Ac & 1.70 & $\mathrm{Ab}$ & 2.10 & $\mathrm{Aa}$ \\
\hline EP-17 & 121.8 & $\mathrm{Aa}$ & 97.3 & $\mathrm{Aa}$ & 2.90 & $\mathrm{Aa}$ & 3.05 & $\mathrm{Aa}$ & 2.53 & $\mathrm{Aa}$ \\
\hline SF-11 & 120.1 & $\mathrm{Aa}$ & 101.2 & $\mathrm{Aa}$ & 2.69 & $\mathrm{Aa}$ & 2.55 & $\mathrm{Aa}$ & 2.30 & $\mathrm{Aa}$ \\
\hline P-294 & 97.5 & $\mathrm{Aa}$ & 59.2 & $\mathrm{Bb}$ & 2.24 & $\mathrm{Ab}$ & 2.33 & $\mathrm{Ab}$ & 2.13 & $\mathrm{Aa}$ \\
\hline P-288 & 96.9 & $\mathrm{Aa}$ & 62.4 & $\mathrm{Bb}$ & 1.87 & $\mathrm{Bc}$ & 1.97 & $\mathrm{Bb}$ & 2.57 & $\mathrm{Aa}$ \\
\hline BRS 506 X 2506 & 76.6 & $\mathrm{Ab}$ & 63.3 & $\mathrm{Ab}$ & 2.11 & $\mathrm{Ab}$ & 2.35 & $\mathrm{Ab}$ & 2.37 & $\mathrm{Aa}$ \\
\hline $2502 \times 467$ & 98.5 & $\mathrm{Aa}$ & 65.2 & $\mathrm{Bb}$ & 2.26 & $\mathrm{Ab}$ & 2.13 & $\mathrm{Ab}$ & 2.39 & $\mathrm{Aa}$ \\
\hline Theis X 2501 & 67.2 & $\mathrm{Ab}$ & 42.1 & $\mathrm{Ab}$ & 1.75 & $\mathrm{Bc}$ & 2.17 & $\mathrm{Bb}$ & 2.69 & $\mathrm{Aa}$ \\
\hline IPA 2564 & 70.0 & $\mathrm{Ab}$ & 79.6 & $\mathrm{Aa}$ & 1.28 & $\mathrm{Bc}$ & 2.08 & $\mathrm{Ab}$ & 2.66 & $\mathrm{Aa}$ \\
\hline BRS Ponta Negra & 94.1 & $\mathrm{Aa}$ & 79.8 & $\mathrm{Aa}$ & 2.16 & $\mathrm{Ab}$ & 2.30 & $\mathrm{Ab}$ & 2.49 & $\mathrm{Aa}$ \\
\hline BRS 655 & 64.1 & $\mathrm{Ab}$ & 69.6 & $\mathrm{Ab}$ & 1.38 & $\mathrm{Bc}$ & 2.16 & $\mathrm{Ab}$ & 2.42 & $\mathrm{Aa}$ \\
\hline Volumax & 83.6 & $\mathrm{Aa}$ & 80.8 & $\mathrm{Aa}$ & 1.51 & $\mathrm{Bc}$ & 2.25 & $\mathrm{Ab}$ & 2.56 & $\mathrm{Aa}$ \\
\hline BRS 658 & 58.5 & $\mathrm{Ab}$ & 74.1 & $\mathrm{Aa}$ & 1.60 & Ac & 2.25 & $\mathrm{Ab}$ & 2.21 & $\mathrm{Aa}$ \\
\hline BRS 659 & 81.0 & $\mathrm{Ab}$ & 68.5 & $\mathrm{Ab}$ & 1.58 & Ac & 2.15 & $\mathrm{Ab}$ & 2.18 & $\mathrm{Aa}$ \\
\hline SF-15 & 95.9 & $\mathrm{Aa}$ & 84.0 & $\mathrm{Aa}$ & 2.69 & $\mathrm{Aa}$ & 2.82 & $\mathrm{Aa}$ & 2.94 & $\mathrm{Aa}$ \\
\hline Means & 80.3 & & 68.8 & & 1.86 & & 2.27 & & 2.47 & \\
\hline CV\% & 18.6 & & 22.31 & & 7.42 & & 13.72 & & 22.87 & \\
\hline
\end{tabular}

*Means in columns followed by the same lowercase letters are not significantly different; means in rows, within traits, followed by the same uppercase letter are not significantly different according to Scott-Knott mean test at $\mathrm{p}<0.05$.

of 94 to 98.5 t of GM per hectare. Among grain sorghum genotypes (Table 3), GM mean values showed much less expressive production, but since the experiment comprised a heterogeneous group of genotypes, the mean GM yield varied from $65.3 \mathrm{t} \mathrm{ha}^{-1}$ in SC971 to $27.6 \mathrm{t} \mathrm{ha}^{-1}$ in ICSV400. As reported by Santos et al. (2013), GM yield with no irrigation was $89.4 \mathrm{t} \mathrm{ha}^{-1}$ (BRS 506), $76.2 \mathrm{t}$ ha $^{-1}$ (BRS Ponta Negra), and 68.0 (BRS 601) t $\mathrm{ha}^{-1}$, while GM yield presented by Perazzo et al. (2013) was $52.1 \mathrm{t} \mathrm{ha}^{-1}$ (BRS Ponta Negra), 43.6 $\mathrm{t} \mathrm{ha}^{-1}$ (IPA 1011), and $42.7 \mathrm{t} \mathrm{ha}^{-1}$ (SF 15). 
Table 3. Means of green matter and plant height for 25 grain sorghum genotypes (E2) evaluated in Petrolina, PE (C.E.B) and in Nossa Senhora da Glória, SE (C.E.G.).

\begin{tabular}{|c|c|c|c|c|c|c|c|c|c|c|}
\hline \multirow{3}{*}{$\begin{array}{c}\text { Genotypes } \\
\text { Sureno } 160.0\end{array}$} & \multicolumn{4}{|c|}{ Green Matter $\left(\mathrm{t} \mathrm{ha}^{-1}\right)^{*}$} & \multicolumn{6}{|c|}{ Plant Height (m) } \\
\hline & \multicolumn{2}{|c|}{ C.E.B. 2017} & \multicolumn{2}{|c|}{ C.E.B 2018} & \multicolumn{2}{|c|}{ C.E.B. 2017} & \multicolumn{2}{|c|}{ C.E.B 2018} & \multicolumn{2}{|c|}{ C.E.G. 2017} \\
\hline & 48.1 & $\mathrm{Ab}$ & 35.6 & $\mathrm{Ab}$ & 1.48 & $\mathrm{Bc}$ & 1.71 & $\mathrm{Bb}$ & 2.20 & $\mathrm{Aa}$ \\
\hline ICSV400 & 27.6 & Ac & 46.8 & $\mathrm{Aa}$ & 1.44 & $\mathrm{Bc}$ & 1.59 & $\mathrm{Bc}$ & 2.13 & $\mathrm{Aa}$ \\
\hline EBA-3 & 27.6 & Ac & 19.7 & $\mathrm{Ab}$ & 1.36 & $\mathrm{Bc}$ & 1.79 & $\mathrm{Bb}$ & 2.26 & $\mathrm{Aa}$ \\
\hline SC971 & 65.2 & $\mathrm{Ab}$ & 31.0 & $\mathrm{Bb}$ & 1.49 & Ac & 1.49 & Ac & 1.66 & $\mathrm{Ab}$ \\
\hline FC13642 Pink Kafir & 47.1 & $\mathrm{Ab}$ & 27.9 & $\mathrm{Ab}$ & 1.38 & $\mathrm{Bc}$ & 1.36 & $\mathrm{Bc}$ & 2.43 & $\mathrm{Aa}$ \\
\hline Marupantse & 49.6 & $\mathrm{Ab}$ & 28.1 & $\mathrm{Ab}$ & 1.54 & Ac & 1.46 & Ac & 1.86 & $\mathrm{Ab}$ \\
\hline PI48770 White Kafir & 42.2 & $\mathrm{Ab}$ & 30.6 & $\mathrm{Ab}$ & 1.25 & $\mathrm{Bc}$ & 1.41 & $\mathrm{Bc}$ & 1.83 & $\mathrm{Ab}$ \\
\hline M35-1 & 53.4 & $\mathrm{Ab}$ & 14.6 & $\mathrm{Bb}$ & 2.02 & $\mathrm{Ab}$ & 1.85 & $\mathrm{Ab}$ & 1.81 & $\mathrm{Ab}$ \\
\hline $\mathrm{SC} 224$ & 48.9 & $\mathrm{Ab}$ & 36.2 & $\mathrm{Ab}$ & 1.71 & $\mathrm{Ac}$ & 1.61 & $\mathrm{Ac}$ & 1.80 & $\mathrm{Ab}$ \\
\hline SC1337 & 59.0 & $\mathrm{Ab}$ & 20.1 & $\mathrm{Bb}$ & 1.29 & $\mathrm{Bc}$ & 1.35 & $\mathrm{Bc}$ & 2.05 & $\mathrm{Aa}$ \\
\hline SC323 & 44.2 & $\mathrm{Ab}$ & 30.6 & $\mathrm{Ab}$ & 1.32 & $\mathrm{Bc}$ & 1.58 & $\mathrm{Bc}$ & 2.24 & $\mathrm{Aa}$ \\
\hline Segaolane & 55.4 & $\mathrm{Ab}$ & 29.0 & $\mathrm{Bb}$ & 1.32 & $\mathrm{Bc}$ & 1.44 & $\mathrm{Bc}$ & 2.00 & $\mathrm{Ab}$ \\
\hline Sureno 167.5 & 43.5 & $\mathrm{Ab}$ & 34.7 & $\mathrm{Ab}$ & 1.45 & Ac & 1.95 & $\mathrm{Ab}$ & 1.82 & $\mathrm{Ab}$ \\
\hline CMSXS189 & 64.7 & $\mathrm{Ab}$ & 63.3 & $\mathrm{Aa}$ & 1.44 & $\mathrm{Bc}$ & 2.04 & $\mathrm{Ab}$ & 1.83 & $\mathrm{Ab}$ \\
\hline SC720 & 45.2 & $\mathrm{Ab}$ & 30.4 & $\mathrm{Ab}$ & 1.06 & $\mathrm{Bc}$ & 1.33 & $\mathrm{Bc}$ & 1.88 & $\mathrm{Ab}$ \\
\hline 9910032 & 59.4 & $\mathrm{Ab}$ & 26.8 & $\mathrm{Bb}$ & 1.19 & $\mathrm{Bc}$ & 1.07 & $\mathrm{Bc}$ & 2.15 & $\mathrm{Aa}$ \\
\hline Pinolero 1 & 52.7 & $\mathrm{Ab}$ & 17.9 & $\mathrm{Bb}$ & 1.29 & $\mathrm{Bc}$ & 1.23 & $\mathrm{Bc}$ & 1.83 & $\mathrm{Ab}$ \\
\hline Jocoro & 29.7 & Ac & 31.7 & $\mathrm{Ab}$ & 1.12 & $\mathrm{Bc}$ & 1.38 & $\mathrm{Bc}$ & 1.76 & $\mathrm{Ab}$ \\
\hline Kuyuma & 30.1 & $\mathrm{Bc}$ & 62.2 & $\mathrm{Aa}$ & 1.10 & $\mathrm{Bc}$ & 1.31 & $\mathrm{Bc}$ & 2.13 & $\mathrm{Aa}$ \\
\hline BRS Ponta Negra & 95.7 & $\mathrm{Aa}$ & 69.6 & $\mathrm{Ba}$ & 2.14 & $\mathrm{Ab}$ & 2.13 & $\mathrm{Ab}$ & 2.20 & $\mathrm{Aa}$ \\
\hline BRS 655 & 83.8 & $\mathrm{Aa}$ & 61.2 & $\mathrm{Aa}$ & 1.41 & $\mathrm{Bc}$ & 1.87 & $\mathrm{Ab}$ & 2.32 & $\mathrm{Aa}$ \\
\hline Volumax & 78.7 & $\mathrm{Aa}$ & 49.0 & $\mathrm{Ba}$ & 1.56 & $\mathrm{Ac}$ & 1.82 & $\mathrm{Ab}$ & 1.89 & $\mathrm{Ab}$ \\
\hline BRS 658 & 65.3 & $\mathrm{Ab}$ & 64.4 & $\mathrm{Aa}$ & 1.64 & Ac & 1.89 & $\mathrm{Ab}$ & 1.94 & $\mathrm{Ab}$ \\
\hline BRS 659 & 57.4 & $\mathrm{Ab}$ & 47.5 & $\mathrm{Aa}$ & 1.59 & Ac & 1.81 & $\mathrm{Ab}$ & 1.91 & $\mathrm{Ab}$ \\
\hline SF-15 & 102.9 & $\mathrm{Aa}$ & 87.8 & $\mathrm{Aa}$ & 3.00 & $\mathrm{Aa}$ & 2.85 & $\mathrm{Aa}$ & 2.04 & $\mathrm{Ba}$ \\
\hline Means & 55.1 & & 39.9 & & 1.50 & & 1.65 & & 2.00 & \\
\hline $\mathrm{CV} \%$ & 26.40 & & 36.61 & & 8.96 & & 12.02 & & 20.24 & \\
\hline
\end{tabular}

** Means in columns followed by the same lowercase letters are not significantly different; means in rows, within traits, followed by the same uppercase letter are not significantly different according to Scott-Knott mean test at $\mathrm{p}<0.05$.

The obtained results can be compared livestock production is an important activity for with those reported by Pimentel et al. (2017), northeastern farmers, the results of GM presented when testing biomass sorghum BD 7607 and in Table 2 and 3 provide important information BRS 716, which produced 110 and $108 \mathrm{tha}^{-1}$ of regarding the potential for forage production $\mathrm{GM}$, corresponding to DM yield of 43 and $41 \mathrm{t}$ under irrigation of experimental and commercial ha $^{-1}$, respectively, in a five-month period. Since sorghum genotypes. 
ANOVA for GM from experiment 1 and 2 (Table 4) showed the magnitude of the variation for this trait with highly significant mean squares (MS) for genotypes (G) and environments (E). Although the $\mathrm{G} x$ E interaction had no significant MS in experiment 1 (forage sorghum genotypes), it was highly significant in experiment 2 (grain sorghum genotypes). Ferrari Júnior et al. (2005) emphasize the importance of taking GM estimates as a key parameter for dimensioning of silos, aiming at silage production.

Plant height $(\mathrm{PH})$ means are presented in Tables 2 and 3 and show taller plants in experiment 1 , with overall mean of $2.20 \mathrm{~m}$, while in experiment 2, they had mean value of $1.72 \mathrm{~m}$. The estimated coefficients of correlation (Table 5) between PH x GM and PH x DM were highly significant, with values of $84.84 \%$ and $57.25 \%$, respectively, thus showing that GM and DM yield are directly and positively influenced by
$\mathrm{PH}$ and determine the architecture of forage and grain sorghum cultivars.

The PH reported by Oliveira et al. (2018) for rainfed sorghum cultivated in Nossa Senhora da Glória, SE, was $3.0 \mathrm{~m}$ for SF-15, BRS 601 and BRS 506; $2.5 \mathrm{~m}$ for BRS 655; and $1.9 \mathrm{~m}$ for IPA 2502. Still in regard to rainfed sorghum, Santos et al. (2013) reported PH for BRS 506 (2.6 m), BRS 601 (2.5 m), BRS Ponta Negra $(2.3 \mathrm{~m})$, BRS $610(2.10 \mathrm{~m})$ and BRS $655(1.9 \mathrm{~m})$, and presented correlation coefficient of 89 and $78 \%$ between PH x GM and PH x DM, respectively. Highly correlated with forage yield (GM and $\mathrm{DM})$, the $\mathrm{PH}$ of irrigated sorghum plants, in this experiment, showed more expressive magnitudes than of those not irrigated. Among the experiments, the most expressive $\mathrm{PH}$ was noticed in 2018, for genotypes 13F03(1141562) and EB 17, with values of 3.08 and $3.05 \mathrm{~m}$, respectively. The joint ANOVA for PH (Table 4)

Table 4. Mean squares (MS), significances and p-values from joint ANOVA of forage (E1) and grain (E2) sorghum experiments, for green matter (GM) and for plant height (PH) from two and three locations, respectively.

\begin{tabular}{|c|c|c|c|c|c|c|c|c|c|c|}
\hline \multirow{3}{*}{ Sources } & \multicolumn{5}{|c|}{ Green Matter } & \multicolumn{5}{|c|}{ Plant Height } \\
\hline & \multicolumn{2}{|r|}{ E1 } & \multicolumn{3}{|c|}{ E2 } & \multicolumn{3}{|c|}{ E1 } & \multicolumn{2}{|l|}{ E2 } \\
\hline & $\mathrm{df}$ & MS. $10^{6}$ & $p$ & MS. $10^{6}$ & $p$ & $\mathrm{df}$ & MS & $p$ & MS & $p$ \\
\hline Genotypes & 24 & 1574.66 & $0.0 * *$ & 1758.66 & $0.0 * *$ & 24 & 0.760 & $0.0 * *$ & 0.597 & $0.0 * *$ \\
\hline Environments & 1 & 5006.30 & $0.0 * *$ & 8702.91 & $0.0 * *$ & 2 & 7.332 & $0.0 * *$ & 4.836 & $0.0 * *$ \\
\hline $\mathrm{G} \times \mathrm{E}$ & 24 & 415.63 & $0.07^{\mathrm{ns}}$ & 437.25 & $0,01 * *$ & 48 & 0.280 & $0.0 * *$ & 0.210 & $0.0 * *$ \\
\hline Error & 98 & 269.46 & & 215.10 & & 148 & 0.151 & & 0.076 & \\
\hline Mean & & \multicolumn{2}{|c|}{$74.6 \mathrm{t} \mathrm{ha}^{-1}$} & \multicolumn{2}{|c|}{$47.5 \mathrm{t} \mathrm{ha}^{-1}$} & \multicolumn{3}{|c|}{$2.20 \mathrm{~m}$} & \multicolumn{2}{|c|}{$1.72 \mathrm{~m}$} \\
\hline $\mathrm{CV} \%$ & & \multicolumn{2}{|c|}{22.02} & \multicolumn{2}{|c|}{30.89} & \multicolumn{3}{|c|}{17.67} & \multicolumn{2}{|c|}{16.02} \\
\hline
\end{tabular}

***: Significant at $1 \%$ and $5 \%$ probability, respectively, according to F-test. 
Table 5. Mean Pearson's correlation coefficients between studied variables, respective significance, and $p$-value.

\begin{tabular}{|c|c|c|c|c|c|c|}
\hline & GM & $\mathrm{PH}$ & DM & GY & SM & PL \\
\hline $\mathrm{PH}$ & $\begin{array}{l}0.8484 * * \\
0.00\end{array}$ & & & & & \\
\hline DM & $\begin{array}{l}0.6829 * * \\
0.00\end{array}$ & $\begin{array}{l}0.5725^{* *} \\
0.00\end{array}$ & & & & \\
\hline GY & $\begin{array}{l}0.0504^{\mathrm{ns}} \\
0.73\end{array}$ & $\begin{array}{l}0.0340^{\mathrm{ns}} \\
0.81\end{array}$ & $\begin{array}{l}-0.20933^{\mathrm{ns}} \\
0.14\end{array}$ & & & \\
\hline SM & $\begin{array}{l}-0.3714 * * \\
0.01\end{array}$ & $\begin{array}{l}-0.2648^{\mathrm{ns}} \\
0.06\end{array}$ & $\begin{array}{l}-0.3962 * * \\
0,00\end{array}$ & $\begin{array}{l}0.2631^{\mathrm{ns}} \\
0.06\end{array}$ & & \\
\hline PL & $\begin{array}{l}0.3645^{* *} \\
0.01\end{array}$ & $\begin{array}{l}0.2076^{\mathrm{ns}} \\
0.14\end{array}$ & $\begin{array}{l}0.4530 * * \\
0.00\end{array}$ & $\begin{array}{l}-0.0835^{\mathrm{ns}} \\
0.57\end{array}$ & $\begin{array}{l}-0.2966^{*} \\
0.03\end{array}$ & \\
\hline FLO & $\begin{array}{l}0.1747^{\mathrm{ns}} \\
0.22\end{array}$ & $\begin{array}{l}0.1825^{\text {ns }} \\
0.20\end{array}$ & $\begin{array}{l}0.3261 * \\
0.02\end{array}$ & $\begin{array}{l}0.2285^{\text {ns }} \\
0.11\end{array}$ & $\begin{array}{l}-0.3269^{*} \\
0.02\end{array}$ & $\begin{array}{l}0.4654 * * \\
0.00\end{array}$ \\
\hline
\end{tabular}

$* * *$ : Significant at $1 \%$ and $5 \%$ probability, respectively, according to t-test.

showed highly significant MS for all sources of variation in both experiments, indicating large variation for this trait, due to the remarkably diverse group of genotypes assessed and different environments.

Mean values for DM, GY and SM were evaluated in 2017 (Tables 6 and 7). Data from DM demonstrated the potential of the forage sorghum genotypes to produce roughage, under drip-irrigation, for ruminant's consumption in the caatinga environment. DM means were highly correlated with GM means (68.29\%) (Table 7) and expressive performance was noted for SF-11 and 13F03(1141562), with values of 45.48 and $39.18 \mathrm{t} \mathrm{ha}^{-1}$, respectively. Santos et al. (2013) reported DM yield ranging from $25.2 \mathrm{t} \mathrm{ha}^{-1}$ for BRS 506 to $17.1 \mathrm{t} \mathrm{ha}^{-1}$ for BRS 655 , with no irrigation. On the other hand, GY, assessed through panicle protection (screen bag) to prevent bird damage, showed expressive grain production in experiment 2, reaching 13.39 and $10.28 \mathrm{t} \mathrm{ha}^{-1}$ in genotypes 9910032 and BRS Ponta Negra, respectively. Grain yield of such magnitude in that semi-arid environment can be attributed to the $\mathrm{C} 4$ photosynthesis pathway of sorghum plants combined with an abundance of solar radiation characteristic of the low latitude of the region where the experimental field is located, in addition to the good soil fertility in the area and full ET replacement irrigation. Zwirtes et al. (2015) observed GY of $6.28 \mathrm{t} \mathrm{ha}^{-1}$ in Santa Maria, RS, while Farre and Faci (2006) reported GY of $8.54 \mathrm{tha}^{-1}$ in Spain, both with replacement of $100 \%$ of ETc. Araya et al. (2018) observed GY for irrigated sorghum in Kansas, USA, of 7.7, 8.4 and $9.3 \mathrm{tha}^{-1}$ in the years of 2008, 2009 and 2012, respectively.

The ANOVA for DM and GY data is presented in Table 8 and Table 9, and showed large variation among the genotypes in both 
Table 6. Means for dry matter, grain yield, seed mass, panicle length and days to flowering of 25 forage sorghum genotypes (E1) evaluated in Petrolina, PE and Nossa Senhora da Glória, SE.

\begin{tabular}{|c|c|c|c|c|c|c|c|c|c|c|c|c|}
\hline \multirow{2}{*}{ Genotypes } & \multicolumn{2}{|c|}{$\begin{array}{c}\text { Dry Matter } \\
\left(\mathrm{t} \mathrm{ha}^{-1}\right)\end{array}$} & \multicolumn{2}{|c|}{$\begin{array}{c}\text { Grain Yield } \\
\left(\mathrm{kg} \mathrm{ha}^{-1}\right)\end{array}$} & \multicolumn{2}{|c|}{$\begin{array}{l}\text { Seed Mass } \\
(\mathrm{mg})\end{array}$} & \multicolumn{2}{|c|}{$\begin{array}{c}\text { Panicle } \\
\text { Length }(\mathrm{cm})\end{array}$} & \multicolumn{4}{|c|}{$\begin{array}{c}\text { Days to } \\
\text { Flowering }\end{array}$} \\
\hline & & & \multicolumn{2}{|c|}{ C.E.B. 2017} & & & \multicolumn{2}{|c|}{ C.E.G 2016} & \multicolumn{2}{|c|}{ C.E.B. 2017} & \multicolumn{2}{|c|}{ C.E.G. 2016} \\
\hline BRS 506 & 37.4 & $\mathrm{a}$ & 3,109 & $\mathrm{c}$ & 27.3 & $\mathrm{c}$ & 21.4 & $\mathrm{c}$ & 64 & $\mathrm{~b}$ & 64 & $\mathrm{e}$ \\
\hline 13F03(1141562) & 39.1 & $\mathrm{a}$ & 5,768 & $\mathrm{~b}$ & 34.0 & $\mathrm{a}$ & 23.5 & $\mathrm{c}$ & 55 & $\mathrm{~d}$ & 63 & $\mathrm{e}$ \\
\hline 13F03(1141572) & 17.7 & $\mathrm{~b}$ & 4,816 & $\mathrm{c}$ & 36.0 & $\mathrm{a}$ & 26.5 & $\mathrm{~b}$ & 54 & $\mathrm{~d}$ & 67 & $\mathrm{~d}$ \\
\hline 947216 & 29.9 & $\mathrm{~b}$ & 5,432 & $\mathrm{~b}$ & 28.3 & $\mathrm{c}$ & 23.1 & $\mathrm{c}$ & 50 & $\mathrm{f}$ & 53 & $f$ \\
\hline 13F02(1141570) & 29.0 & $\mathrm{~b}$ & 9,464 & $\mathrm{a}$ & 30.3 & $\mathrm{~b}$ & 23.9 & $\mathrm{c}$ & 61 & $\mathrm{c}$ & 66 & $\mathrm{e}$ \\
\hline 9929030 & 23.0 & $\mathrm{~b}$ & 2,008 & $\mathrm{c}$ & 26.0 & $\mathrm{c}$ & 22.9 & $\mathrm{c}$ & 48 & $\mathrm{f}$ & 53 & $f$ \\
\hline 947030 & 22.3 & $\mathrm{~b}$ & 3,104 & $\mathrm{c}$ & 30.7 & $\mathrm{~b}$ & 23.3 & $\mathrm{c}$ & 51 & $\mathrm{e}$ & 53 & $\mathrm{f}$ \\
\hline 947254 & 25.0 & $\mathrm{~b}$ & 6,736 & $\mathrm{~b}$ & 23.3 & $\mathrm{~d}$ & 21.9 & $\mathrm{c}$ & 55 & $\mathrm{~d}$ & 63 & $\mathrm{e}$ \\
\hline SS318 & 28.4 & $\mathrm{~b}$ & 3,517 & $\mathrm{c}$ & 30.7 & $\mathrm{~b}$ & 25.1 & $\mathrm{c}$ & 51 & $\mathrm{e}$ & 54 & $\mathrm{f}$ \\
\hline ICSV745 & 27.2 & $\mathrm{~b}$ & 7,504 & $\mathrm{a}$ & 24.7 & $\mathrm{~d}$ & 22.8 & $\mathrm{c}$ & 62 & $\mathrm{c}$ & 67 & $\mathrm{~d}$ \\
\hline BRS Ponta Negra & 31.3 & $\mathrm{a}$ & 9,168 & $\mathrm{a}$ & 31.7 & $\mathrm{~b}$ & 26.1 & $\mathrm{~b}$ & 65 & $\mathrm{~b}$ & 71 & $\mathrm{c}$ \\
\hline IPA 2502 & 12.2 & $\mathrm{~b}$ & 6,914 & $\mathrm{~b}$ & 29.3 & $\mathrm{c}$ & 23.9 & $\mathrm{c}$ & 62 & $\mathrm{c}$ & 63 & $\mathrm{e}$ \\
\hline EP-17 & 37.1 & $\mathrm{a}$ & 4,727 & $\mathrm{c}$ & 20.3 & $\mathrm{~d}$ & 33.9 & $\mathrm{a}$ & 70 & $\mathrm{a}$ & 84 & $\mathrm{a}$ \\
\hline SF-11 & 45.5 & $\mathrm{a}$ & 2,801 & $\mathrm{c}$ & 21.3 & $\mathrm{~d}$ & 28.5 & $\mathrm{~b}$ & 71 & $\mathrm{a}$ & 80 & $\mathrm{a}$ \\
\hline P-294 & 29.4 & $\mathrm{~b}$ & 4,712 & $\mathrm{c}$ & 17.3 & $\mathrm{e}$ & 28.7 & $\mathrm{~b}$ & 62 & $\mathrm{c}$ & 68 & $\mathrm{~d}$ \\
\hline P-288 & 26.2 & $\mathrm{~b}$ & 5,512 & $\mathrm{~b}$ & 20.3 & d & 25.9 & $\mathrm{~b}$ & 70 & $\mathrm{a}$ & 74 & $\mathrm{~b}$ \\
\hline BRS 506 X 2506 & 24.8 & $\mathrm{~b}$ & 7,899 & $\mathrm{a}$ & 20.7 & $\mathrm{~d}$ & 19.1 & $\mathrm{c}$ & 53 & $\mathrm{~d}$ & 53 & $f$ \\
\hline $2502 \times 467$ & 36.6 & $\mathrm{a}$ & 5,368 & $\mathrm{~b}$ & 20.3 & $\mathrm{~d}$ & 24.7 & $\mathrm{c}$ & 54 & $\mathrm{~d}$ & 58 & $f$ \\
\hline Theis X 2501 & 27.0 & $\mathrm{~b}$ & 2,138 & $\mathrm{c}$ & 27.0 & $\mathrm{c}$ & 23.1 & $\mathrm{c}$ & 48 & $\mathrm{f}$ & 53 & $f$ \\
\hline IPA 2564 & 23.3 & $\mathrm{~b}$ & 6,957 & $\mathrm{~b}$ & 29.0 & $\mathrm{c}$ & 20.2 & $\mathrm{c}$ & 54 & $\mathrm{~d}$ & 54 & $f$ \\
\hline BRS 655 & 19.4 & $\mathrm{~b}$ & 5,936 & $\mathrm{~b}$ & 32.7 & $\mathrm{~b}$ & 23.2 & $\mathrm{c}$ & 48 & $f$ & 53 & $f$ \\
\hline Volumax & 34.3 & $\mathrm{a}$ & 2,272 & $\mathrm{c}$ & 34.0 & $\mathrm{a}$ & 25.3 & $\mathrm{c}$ & 51 & $\mathrm{e}$ & 53 & $f$ \\
\hline BRS 658 & 23.8 & $\mathrm{~b}$ & 4,062 & $\mathrm{c}$ & 26.0 & $\mathrm{c}$ & 22.2 & $\mathrm{c}$ & 48 & $f$ & 53 & $f$ \\
\hline BRS 659 & 28.8 & $\mathrm{~b}$ & 8,013 & $\mathrm{a}$ & 31.3 & $\mathrm{~b}$ & 24.6 & $\mathrm{c}$ & 49 & $\mathrm{f}$ & 53 & $f$ \\
\hline SF-15 & 34.4 & $\mathrm{a}$ & 5,588 & $\mathrm{~b}$ & 17.0 & $\mathrm{e}$ & 28.5 & $\mathrm{~b}$ & 71 & $\mathrm{a}$ & 81 & $\mathrm{a}$ \\
\hline Mean & 28.5 & & 5,340 & & 26.8 & & 22.0 & & 57.0 & & 62. & \\
\hline $\mathrm{CV} \%$ & 20.18 & & 29.26 & & 8.53 & & 11.0 & & 2.65 & & 3.44 & \\
\hline
\end{tabular}

** Means in columns followed by the same lowercase letters are not significantly different according to Scott-Knott mean test at $\mathrm{p}<0.05$.

experiments, with highly significant MS for 5), estimated between GM or DM and GY, were G. Overall means of the experiments for DM 5.0 and $-20.93 \%$, respectively, indicating that the and GY corroborates the assumed specialty $\mathrm{x}$ genotypes in experiment 1 produced more forage architecture correlation in the studied cultivar $\left(28.53 \mathrm{t} \mathrm{ha}^{-1}\right)$ than those in experiment $2(20.67 \mathrm{t}$ group. Pearson's correlation coefficients (Table ha ${ }^{-1}$ ) in detriment of GY, and inversely, genotypes 
Table 7. Means for dry matter, grain yield, seed mass, panicle length and days to flowering of 25 grain type sorghum genotypes (E2) evaluated in Petrolina, PE and Nossa Senhora da Glória, SE.

\begin{tabular}{|c|c|c|c|c|c|c|c|c|c|c|c|c|}
\hline \multirow{2}{*}{ Genotypes } & \multirow{2}{*}{\multicolumn{2}{|c|}{$\begin{array}{c}\text { Dry Matter } \\
\left(\mathrm{t} \mathrm{ha}^{-1}\right)\end{array}$}} & \multirow{2}{*}{\multicolumn{2}{|c|}{$\begin{array}{c}\begin{array}{c}\text { Grain Yield } \\
\left(\mathrm{kg} \mathrm{ha}^{-1}\right)\end{array} \\
\text { C.E.B. } 2017\end{array}$}} & \multirow{2}{*}{\multicolumn{2}{|c|}{$\begin{array}{l}\text { Seed Mass } \\
(\mathrm{mg})\end{array}$}} & \multirow{2}{*}{\multicolumn{2}{|c|}{$\begin{array}{c}\text { Panicle } \\
\text { Length }(\mathrm{cm})\end{array}$}} & \multicolumn{4}{|c|}{$\begin{array}{c}\text { Days to } \\
\text { Flowering }\end{array}$} \\
\hline & & & & & & & & & C.E.B. 20 & & C.E.G. 2 & \\
\hline Sureno 160.0 & 17.3 & $\mathrm{~b}$ & 6,427 & $\mathrm{c}$ & 28.0 & $\mathrm{c}$ & 18.87 & $\mathrm{c}$ & 58 & $\mathrm{c}$ & 64 & $\mathrm{c}$ \\
\hline ICSV400 & 9.7 & $\mathrm{~b}$ & 9,016 & $\mathrm{c}$ & 37.0 & $\mathrm{a}$ & 23.33 & $\mathrm{~b}$ & 60 & $\mathrm{c}$ & 63 & $\mathrm{c}$ \\
\hline EBA-3 & 12.1 & $\mathrm{~b}$ & 3,588 & d & 39.0 & $\mathrm{a}$ & 16.00 & d & 52 & $\mathrm{~d}$ & 54 & d \\
\hline SC971 & 25.6 & $\mathrm{a}$ & 4,293 & $\mathrm{~d}$ & 23.7 & $\mathrm{c}$ & 18.47 & $\mathrm{c}$ & 51 & $\mathrm{~d}$ & 56 & $\mathrm{~d}$ \\
\hline FC13642 Pink Kafir & 20.7 & $\mathrm{~b}$ & 3,508 & $\mathrm{~d}$ & 28.3 & $\mathrm{c}$ & 24.33 & $\mathrm{~b}$ & 53 & $\mathrm{~d}$ & 53 & $\mathrm{~d}$ \\
\hline Marupantse & 18.2 & $\mathrm{~b}$ & 4,247 & $\mathrm{~d}$ & 35.3 & $\mathrm{a}$ & 26.47 & $\mathrm{a}$ & 52 & $\mathrm{~d}$ & 53 & $\mathrm{~d}$ \\
\hline PI48770 White Kafir & 17.6 & $\mathrm{~b}$ & 5,062 & $\mathrm{~d}$ & 31.0 & $\mathrm{~b}$ & 20.53 & $\mathrm{~b}$ & 51 & $\mathrm{~d}$ & 53 & $\mathrm{~d}$ \\
\hline M35-1 & 21.3 & $\mathrm{~b}$ & 5,348 & $\mathrm{~d}$ & 36.7 & $\mathrm{a}$ & 14.60 & $d$ & 61 & $\mathrm{c}$ & 75 & $\mathrm{a}$ \\
\hline $\mathrm{SC} 224$ & 18.4 & $\mathrm{~b}$ & 2,781 & $\mathrm{~d}$ & 17.7 & $\mathrm{~d}$ & 25.27 & $\mathrm{a}$ & 49 & $\mathrm{e}$ & 54 & $\mathrm{~d}$ \\
\hline SC1337 & 23.1 & $\mathrm{a}$ & 1,585 & d & 35.3 & $\mathrm{a}$ & 23.40 & $\mathrm{~b}$ & 44 & e & 53 & $\mathrm{~d}$ \\
\hline $\mathrm{SC} 323$ & 19.0 & $\mathrm{~b}$ & 4,396 & $\mathrm{~d}$ & 25.3 & $\mathrm{c}$ & 13.33 & $d$ & 48 & e & 53 & $\mathrm{~d}$ \\
\hline Segaolane & 22.9 & $\mathrm{a}$ & 4,956 & $\mathrm{~d}$ & 31.0 & $\mathrm{~b}$ & 22.20 & $\mathrm{~b}$ & 48 & e & 53 & $\mathrm{~d}$ \\
\hline Sureno 167.5 & 16.2 & $\mathrm{~b}$ & 6,325 & $\mathrm{c}$ & 28.3 & $\mathrm{c}$ & 19.07 & $\mathrm{c}$ & 60 & $\mathrm{c}$ & 63 & $\mathrm{c}$ \\
\hline CMSXS189 & 29.5 & $\mathrm{a}$ & 7,045 & $\mathrm{c}$ & 30.3 & $\mathrm{~b}$ & 17.73 & $\mathrm{c}$ & 51 & d & 53 & $\mathrm{~d}$ \\
\hline SC720 & 15.1 & $\mathrm{~b}$ & 6,083 & $\mathrm{~d}$ & 27.0 & $\mathrm{c}$ & 15.73 & $d$ & 48 & e & 54 & $\mathrm{~d}$ \\
\hline 9910032 & 18.0 & $\mathrm{~b}$ & 13,393 & $\mathrm{a}$ & 39.0 & $\mathrm{a}$ & 23.07 & $\mathrm{~b}$ & 54 & $\mathrm{~d}$ & 64 & $\mathrm{c}$ \\
\hline Pinolero 1 & 16.2 & $\mathrm{~b}$ & 7,905 & $\mathrm{c}$ & 35.7 & $\mathrm{a}$ & 22.27 & $\mathrm{~b}$ & 58 & $\mathrm{c}$ & 64 & $\mathrm{c}$ \\
\hline Jocoro & 9.6 & $\mathrm{~b}$ & 7,059 & $\mathrm{c}$ & 28.7 & $\mathrm{c}$ & 26.20 & $\mathrm{a}$ & 69 & $\mathrm{a}$ & 63 & $\mathrm{c}$ \\
\hline Kuyuma & 9.0 & $\mathrm{~b}$ & 7,262 & $\mathrm{c}$ & 31.3 & $\mathrm{~b}$ & 20.73 & $\mathrm{~b}$ & 60 & $\mathrm{c}$ & 65 & $\mathrm{c}$ \\
\hline BRS Ponta Negra & 31.6 & $\mathrm{a}$ & 10,285 & $\mathrm{~b}$ & 33.0 & $\mathrm{~b}$ & 23.80 & $\mathrm{~b}$ & 65 & $\mathrm{~b}$ & 70 & $\mathrm{~b}$ \\
\hline BRS 655 & 27.8 & $\mathrm{a}$ & 5,631 & $\mathrm{~d}$ & 28.7 & $\mathrm{c}$ & 22.80 & $\mathrm{~b}$ & 49 & $\mathrm{e}$ & 53 & $\mathrm{~d}$ \\
\hline Volumax & 32.4 & $\mathrm{a}$ & 5,314 & $\mathrm{~d}$ & 36.7 & $\mathrm{a}$ & 24.87 & $\mathrm{a}$ & 54 & $\mathrm{~d}$ & 53 & $\mathrm{~d}$ \\
\hline BRS 658 & 24.3 & $\mathrm{a}$ & 6,692 & $\mathrm{c}$ & 28.7 & $\mathrm{c}$ & 22.93 & $\mathrm{~b}$ & 50 & $\mathrm{e}$ & 53 & $\mathrm{~d}$ \\
\hline BRS 659 & 21.6 & $\mathrm{~b}$ & 7,345 & $\mathrm{c}$ & 31.7 & $\mathrm{~b}$ & 23.47 & $\mathrm{~b}$ & 50 & $\mathrm{~d}$ & 53 & $\mathrm{~d}$ \\
\hline SF-15 & 39.6 & $\mathrm{a}$ & 4,147 & $\mathrm{~d}$ & 19.0 & $\mathrm{~d}$ & 28.60 & $\mathrm{a}$ & 72 & $\mathrm{a}$ & 77 & $\mathrm{a}$ \\
\hline Mean & 20.7 & & 5,987 & & 30.6 & & 24.00 & & 54.7 & & 58.8 & \\
\hline $\mathrm{CV} \%$ & 36.33 & & 28.60 & & 10.97 & & 7.93 & & 4.07 & & 2.99 & \\
\hline
\end{tabular}

** Means in columns followed by the same lowercase letters are not significantly different according to Scott-Knott mean test at $\mathrm{p}<0.05$

in experiment 2 were more efficient as to $\mathrm{GY}$ (5.99 $\left.\mathrm{t} \mathrm{ha}^{-1}\right)$ than in experiment $1\left(5.34 \mathrm{t} \mathrm{ha}^{-1}\right)$, regardless of the forage production.

The SM, PL, and FLO traits (Table 6 and 7) demonstrate the large variation among the studied sorghum genotypes. The mass of one sorghum seed ranged from 17 to $36 \mathrm{mg}$ and from 17.7 to $39 \mathrm{mg}$ in experiment 1 and 2, respectively. Bell et al. (2018) reported SM ranging from 25.4 to 27.9 $\mathrm{mg}$, with no correlation being reported between SM and GY, under full irrigation system. The sorghum inflorescence length was measured at 
harvest and varied from 19.07 to $33.93 \mathrm{~cm}$ and from 14.60 to $26.47 \mathrm{~cm}$ in experiment 1 and 2, respectively. The genotype cycle was assessed through the number of days taken to reach $50 \%$ flowering, with data collected from two distinct environments. Days to flowering, evaluated at C.E.B 2017, ranged from 48 to 71 days and from 44 to 69 days, and at C.E.G 2016, they ranged from 53 to 81 days and from 53 to 75 days, in experiment 1 and 2, respectively. Discrepancies among locations in the experiments are probably due to the geographic position and the fact that irrigation was not provided at C.E.G. Furthermore, the crop cycle varied according to the water availability, which is a known strategy of sorghum plants to escape drought.

In Table 5, the estimated correlation coefficients between FLO or PL and SM were negative and significant, indicating that the bigger the panicle length and the later the flowering, the

Table 8. Genotype mean squares (MS), significances from ANOVA, in forage (E1) and grain (E2) sorghum experiments, for dry matter (DM), grain yield (GY) and seed mass (SM) evaluated at C.E.Bebedouro, 2017.

\begin{tabular}{|c|c|c|c|c|c|c|c|}
\hline \multirow{3}{*}{ Sources } & \multicolumn{3}{|c|}{ Dry Matter } & \multicolumn{2}{|c|}{ Grain Yield } & \multicolumn{2}{|c|}{ Seed Mass } \\
\hline & & E1 & E2 & E1 & E2 & E1 & E2 \\
\hline & $\mathrm{df}$ & MS. $10^{6}$ & MS. $10^{6}$ & MS. $10^{6}$ & MS. $10^{6}$ & MS & MS \\
\hline Genotypes & 24 & $165.045^{* *}$ & $169.815^{* *}$ & $13.864 * *$ & $18.626 * *$ & $0.899 * *$ & $0.941 * *$ \\
\hline Error & 48 & 33.178 & 56.407 & 2.443 & 2.932 & 0,052 & 0,113 \\
\hline Mean & & 28.5 & 20.7 & 5,340 & 5,987 & 26.8 & 30.6 \\
\hline CV\% & & 20.18 & 36.33 & 29.26 & 28.60 & 8.53 & 10.97 \\
\hline
\end{tabular}

**: Significant at $1 \%$ probability according to F-test.

Table 9. Genotype mean squares (MS), significances from ANOVA, in forage (E1) and grain (E2) sorghum cultivars, for days to flowering (FLO) and panicle length (PL).

\begin{tabular}{lccccccc}
\hline & \multicolumn{3}{c}{ C.E.B. 2017 } & \multicolumn{4}{c}{ C.E.G 2016 } \\
\hline \multirow{2}{*}{ Sources } & \multicolumn{2}{c}{ Days to Flowering } & \multicolumn{2}{c}{ Days to Flowering } & \multicolumn{2}{c}{ Panicle Length } \\
\cline { 2 - 8 } & \multicolumn{2}{c}{ E1 } & E2 & E1 & E2 & E1 & E2 \\
\hline \multirow{2}{*}{ Genotypes } & 24 & $185.66^{* *}$ & $143.037^{* *}$ & $296.281^{* *}$ & $168.28^{* *}$ & $46.23^{* *}$ & $29.15^{* *}$ \\
Error & 48 & 2.289 & 4.463 & 4.573 & 3.104 & 5.60 & 3.77 \\
\hline Mean & & 57.02 & 54.71 & 62.17 & 58.84 & $0.22 \mathrm{~m}$ & $0.24 \mathrm{~m}$ \\
CV\% & 2.65 & 4.07 & 3.44 & 2.99 & 11.00 & 7.93 \\
\hline
\end{tabular}

**: Significant at $1 \%$ probability according to F-test. 
smaller the seeds are, while a highly significant coefficient of $46.54 \%$ was found between FLO and PL, indicating an important panicle development before blooming.

Genotypes can be tailored, according to the purpose of use, through conventional breeding. The best forage cultivars had the tallest plants and open panicle (SF-11 and SF-15), while grain cultivars were considerably shorter and had compact panicle. Double purpose genotypes tend to combine those traits as it can be seen in BRS Ponta Negra, with intermediate plant height and compact panicle.

\section{Conclusions}

Sorghum plants are fully adapted to the semi-arid environment and the grain and forage production of sorghum cultivars are not limited by temperature during plant development.

The water supply through drip irrigation provides grain and forage yield at outstanding levels in the semi-arid environment.

The genotypes with the best performance as to GM production are EP 17, SF 11, BRS 506 and SF 15, while SF-11, SF-15, 13F03(1141562), BR 506 and EP-17 are the most productive in regard to DM.

The genotypes 9910032, BRS Ponta Negra and ICVS 400 present distinguishable grain yield superior to $10 \mathrm{tha}^{-1}$.

\section{Acknowledgments}

To the Brazilian Agricultural Research Corporation - Embrapa (SEG 23.13.06.014.00.00).

\section{References}

ARAYA, A.; KISEKKA, I.; GOWDA, P. H.; PRASAD, P. V. V. Grain sorghum production functions under different irrigation capacities. Agricultural Water Management, v. 203, p. 261-271, 2018. DOI: 10.1016/j. agwat.2018.03.010.

BELL, J. M.; SCHWARTZ, R.; McINNES, K. J.; HOWELL, T.; MORGAN, C. L. S. Deficit irrigation effects on yield and yield components of grain sorghum. Agricultural Water Management, v. 203, p. 289-296, 2018. DOI: 10.1016/j.agwat.2018.03.002.

FARRE, I.; FACI, J. M. Comparative response of maize (Zea mays L.) and sorghum (Sorghum bicolor L. Moench) to deficit irrigation in a Mediterranean environment. Agricultural Water Management, v. 83, n. 1/2, p. 135-143, 2006. DOI: 10.1016/j.agwat.2005.11.001.

FERRARI JÚNIOR, E.; POSSENTI, R. A.; LIMA, M. L.P.; NOGUEIRA, J.R.;ANDRADE, J. B. Características, composição química e qualidade de silagens de oito cultivares de milho. Boletim de Indústria Animal, v. 62, p. 
19-27, 2005.

IBGE. Sistema IBGE de Recuperação Automática - SIDRA. Pesquisa da pecuária municipal 2019: tabela 3939: efetivo dos rebanhos, por tipo de rebanho e grande região, 1974 a 2018. [Rio de Janeiro, 2019]. Available in: <https://sidra.ibge. gov.br/tabela/3939>. Access in: 25 set. 2019.

MAGALHÃES， P. C.; DURÃES，F. O. M.; RODRIGUES, J. A. S. Fisiologia da planta de sorgo. Sete Lagoas: Embrapa Milho e Sorgo, 2003. 4 p. (Embrapa Milho e Sorgo. Comunicado Técnico, 86).

OLIVEIRA, B. S.; PEREIRA, L. G. R.; AZEVÊDO, J. A. G.; RODRIGUES, J. A. S.; VELASCO, F. O.; NEVES,A. L.A.; MAURÍCIO, R. M.; VERNEQUE, R. da S.; SANTOS, R. D. Silage quality of six sorghum cultivars for sheep. Pesquisa Agropecuária Brasileira, v. 53, n. 2, p. 256-264, 2018. DOI: 10.1590/s0100$204 \times 2018000200015$.

PERAZZO, A. F.; SANTOS, E. M.; PINHO, R. M. A.; CAMPOS, F. S.; RAMOS, J. P. F.; AQUINO, M. M.; SILVA, T. C.; BEZERRA, H. F. C. Agronomic characteristics and efficiency of rainwater use in sorghum cultivars in the semi-arid region. Ciência Rural, v. 43, n. 10, p. 1771-1776, 2013. DOI: 10.1590/S010384782013001000007.

PIMENTEL, L. D.; BATISTA, V. A. P.;
BARROS, A. F. de; TEÓFILO, R. F.; DIAS, L. A. dos S. Chemical and bioenergetic characterization of sorghum agronomic groups.

Pesquisa

Agropecuária

Tropical, v. 47, n. 4, p. 424-431, 2017. DOI: $10.1590 / 1983-40632017 v 4749170$.

SANTOS, F. G.; CASELA, C. R.; WAQUIL, J. M. Melhoramento de sorgo. In: BORÉM, A. (Org.). Melhoramento de espécies cultivadas. 2. ed. Viçosa, MG: Universidade Federal de Viçosa, 2005. p. 429-466.

SANTOS, R. D.; PEREIRA, L. G. R.; NEVES, A. L. A.; RODRIGUES, J. A. S.; COSTA, C. T. F.; OLIVEIRA, G. F. de. Agronomic characteristics of forage sorghum cultivars for silage production in the lower middle San Francisco Valley. Acta Scientiarum - Animal Sciences, v. 35, n. 1, p. 13-19, 2013. DOI: 10.4025/actascianimsci. v35i1.13072.

SILVA, P. C. G. da; MOURA, M. S. B. de; KILL, L. H. P.; BRITO, L. T. de L.; PEREIRA, L. A.; SÁ, I. B.; CORREIA, R. C.; TEIXEIRA, A. H. de C.; CUNHA, T. J. F.; GUIMARÃES FILHO, C. Caracterização do semiárido brasileiro: fatores naturais e humanos. In: SÁ, I. B.; SILVA, P. C. G. da (Ed.). Semiárido brasileiro: pesquisa, desenvolvimento e inovação. Petrolina: Embrapa Semiárido, 2010. p. 18-48. 
ZWIRTES, A. L.; CARLESSO, R.; PETRY, M.

T.; KUNZ, J.; REIMANN, G. K. Desempenho

produtivo e retorno econômico da cultura

do sorgo submetida à irrigação deficitária.

Engenharia Agrícola, v. 35, n. 4, p. 676-688,

2015. DOI: 10.1590/1809-4430-Eng.Agric.

v35n4p676-688/2015. 